Rhode Island College

Digital Commons @ RIC

Master's Theses, Dissertations, Graduate

Master's Theses, Dissertations, Graduate

Research and Major Papers Overview

Research and Major Papers

2017

\title{
What Are the Factors Contributing to the Delay in Hip Fracture Repair?
}

Karen Padraic

Rhode Island College

Follow this and additional works at: https://digitalcommons.ric.edu/etd

Part of the Nursing Commons

\section{Recommended Citation}

Padraic, Karen, "What Are the Factors Contributing to the Delay in Hip Fracture Repair?" (2017). Master's Theses, Dissertations, Graduate Research and Major Papers Overview. 193.

https://digitalcommons.ric.edu/etd/193

This Major Paper is brought to you for free and open access by the Master's Theses, Dissertations, Graduate Research and Major Papers at Digital Commons @ RIC. It has been accepted for inclusion in Master's Theses, Dissertations, Graduate Research and Major Papers Overview by an authorized administrator of Digital Commons @ RIC. For more information, please contact digitalcommons@ric.edu. 


\title{
WHAT ARE THE FACTORS CONTRIBUTING TO THE DELAY IN HIP FRACTURE REPAIR?
}

\author{
by \\ Karen Padraic \\ A Major Paper Submitted in Partial Fulfillment \\ of the Requirements for the Degree of \\ Master of Science in Nursing \\ in \\ The School of Nursing \\ Rhode Island College \\ 2017
}




\begin{abstract}
Hip fracture injuries are identified as one of the most serious healthcare problems affecting older adults (CDC). The prognosis after hip fracture is unfavorable and approximately $25 \%$ of elderly patients will die within one year after the event. Surgical repair is a critical element in the management of a hip fracture. Delay of surgery prolongs hospital stay and increases the risk of medical complications. Despite evidence showing early time to surgery reduces morbidity and mortality for patients with hip fractures, time delays to surgery still exist. The purpose of this quality improvement project was to explore patient- and hospital-specific barriers to early surgery for older adults with hip fractures in a community hospital. Kathleen Stevens' ACE Star Model was used as a conceptual framework to guide this project. A retrospective chart review was conducted with a total of 62 charts consisting of 55 females and 8 males who experienced a hip fracture. Data collected included age, gender, time from emergency room arrival to admission, time from admission to operative intervention, current medications on admission, current use of tobacco and alcohol, type of surgical intervention, and length of stay. Average length of time from admission to surgery was 29 hours. Eight charts were identified with a delay of greater than 48 hours to surgery. Patient-specific barriers were identified. Further research is indicated to determine hospital-specific barriers to early surgery.
\end{abstract}




\section{Table of Contents}

Background and Statement of the Problem ….......................................................... 5







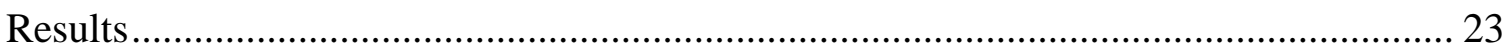

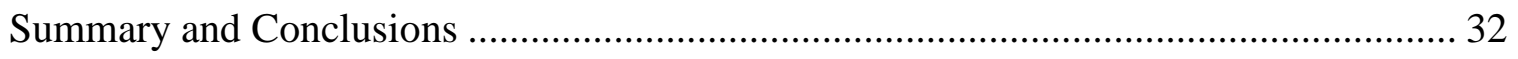

Recommendations and Implications for Practice .................................................... 36

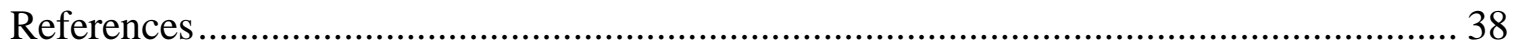

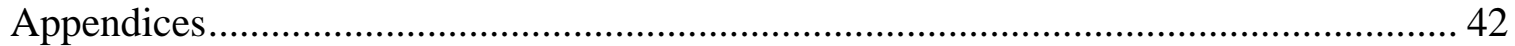




\section{WHAT ARE THE FACTORS CONTRIBUTING TO THE DELAY IN}

\section{HIP FRACTURE REPAIR?}

\section{Background/Statement of the Problem}

The growth in the number and proportion of older adults is unprecedented in the history of the United States (U.S.). Longer life spans and aging baby boomers are two factors that will double the population of Americans aged 65 or older within the next 25 years to 72 million (CDC, 2013). The Center for Disease Control and Prevention (CDC) predicts by 2030 , older adults will account for approximately $20 \%$ of the U.S. population (CDC). Data from The National Report Card on Healthy Aging: How Healthy Are Older Adults in the U.S. show $31.7 \%$ of adults aged 65 or older sustained a fall with injury in the year 2010. According to data from the National Hospital Discharge Survey, 250,000 people aged 65 and older are hospitalized for hip fractures annually. Hip fracture injuries are identified as one of the most serious healthcare problems affecting older adults (CDC). A fractured hip leaves an individual immobile for an extended period of time, thereby slowing circulation, potentiating abnormal clotting and causing increased muscle wasting. These conditions are particularly dangerous for the older adults, being more likely to form clots in their leg veins due to other comorbid conditions such as hypertension or diabetes (Campos et al, 2015).

The prognosis after hip fracture is unfavorable and approximately $25 \%$ of elderly patients will die within one year after the event. Nearly $70 \%$ of patients who survive the acute post-operative stage are discharged to a nursing home. Only $25 \%$ of hip fracture patients regain their former level of independence and the remaining patients sustain 
varying grades of disability. Supportive home care and short or long-term nursing home residence are required. Hip fractures can lead to a significant loss of independence in the community and have a detrimental effect on quality of life (Salkeld, et al, 2000). One in every four hip fracture patients (18-33\%) die within one year of injury (CDC, 2013), further compounding the financial costs of hip fracture. According to the American Academy of Orthopedic Surgeons (AAOS), the economic burden of hip fracture was estimated at \$17-20 billion in 2010 (AAOS, 2014).

Surgical repair is a critical element in the management of a hip fracture. Delay of surgery prolongs hospital stay and increases the risk of pulmonary embolism, deep venous thrombosis, heart failure, urinary tract infection and pressure ulcers. These potential complications may further delay rehabilitation and increase the risk of death (Campos et al., 2015). Early surgery was associated with fewer days of pain and shorter length of hospital stay by 1.94 days (Orosz, et al, 2004). According to the AAOS, moderate evidence exists to support that hip fracture surgery within 48 hours of admission is associated with better patient outcomes. Surgeries delayed due to patient comorbidities had the highest mortality rates and this subset of patient population would benefit the most from earlier surgery (AAOS, 2014).

According to the Minimum Standards for the Management of Hip Fractures in the Older Person (Agency for Clinical Innovation, 2014), patients should be assessed for the presence of correctable comorbidities to ensure surgery is not delayed. Common problematic comorbidities may include anemia, anticoagulation, volume depletion, electrolyte imbalances, uncontrolled diabetes, heart failure, and minor cardiac arrhythmia 
or ischemia (Bottle \& Aylin, 2006).Although many studies have demonstrated delaying surgery for more than 24 hours increases mortality, other studies show no significant difference in mortality risks (Forni et al., 2016).

This research project aimed to explore patient and hospital-specific reasons for delays to surgical hip fracture repair in older adults. Patient outcomes after hip fracture are improved with early hospital admission and surgery. However, there are many preventable delays to admission and surgery. There is a research gap regarding patient and hospital-specific barriers to early hip fracture surgery for older adults in a community hospital setting.

Next, the theoretical framework used to guide this research will be presented. 


\section{Theoretical Framework}

Evidence-based practice (EBP) is the integration of patient values, clinical expertise, and research evidence in the decision making for patient care (Stevens, 2012). The challenge with EBP lies in integrating evidence into practice. The ACE STAR Model of Knowledge Transformation is a framework for systematically operationalizing EBP processes. The model is composed of five major stages: knowledge discovery, evidence summary, translation into practice recommendations, integration into practice, and evaluation (Stevens, 2012) and is illustrated in Figure 1 below.

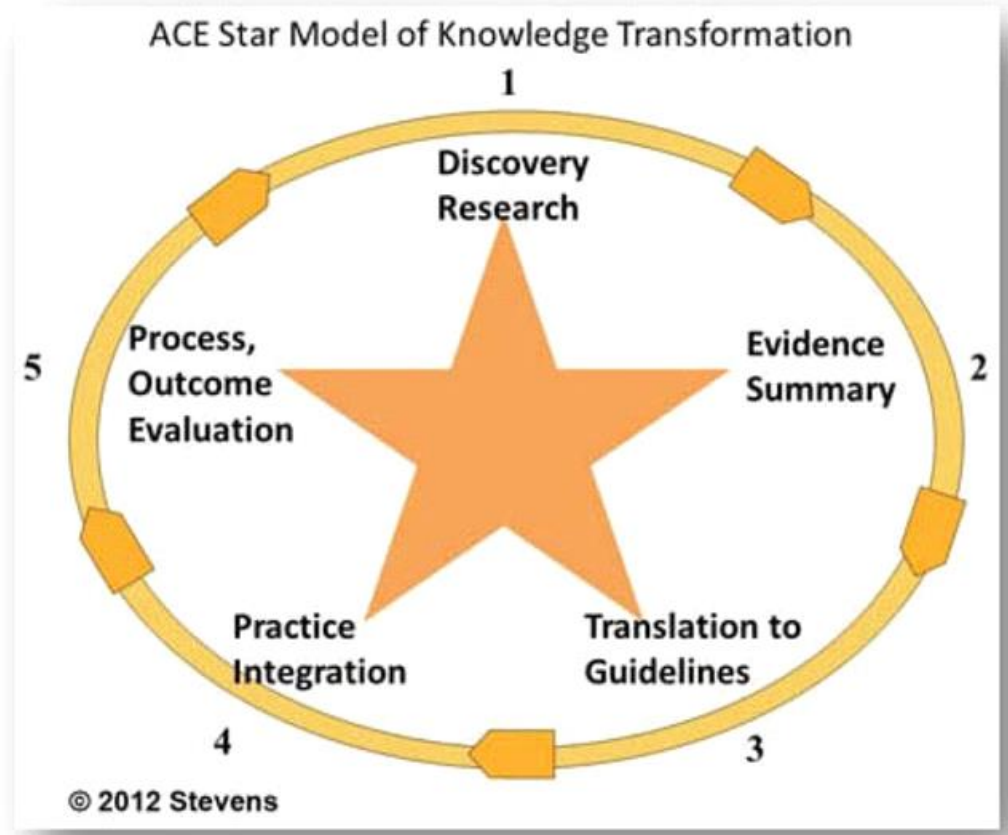

Figure 1. ACE Star Model of Knowledge Transformation

During the research stage, new knowledge is identified through research

methodologies and scientific inquiry. Research results are generated by conducting a single study. This stage builds the main body of research about clinical actions (Stevens, 
2012). The evidence summary or knowledge-generating stage is used to integrate the main body of research knowledge into a single, meaningful statement. New knowledge combines the findings from all studies and identifies bias and limits chance effects in the conclusions. This systematic methodology also increases reliability and reproducibility of results (Stevens, 2012).

The purpose of the translation stage is to provide useful and relevant evidence to clinicians in a form that is appropriate to the time, cost, and care standard. Recommendations are termed clinical practice guidelines and may be represented or embedded in care standards, clinical pathways, protocols, and algorithms. These guidelines are used as tools to support informed clinical decisions for practitioners and the organization (Stevens, 2012). The practice integration stage involves changing both individual and organizational practices. Major aspects addressed are factors that affect individual and organizational rate of adoption of innovation and the integration of the change into sustainable systems (Stevens, 2012).The final stage in knowledge transformation is the impact evaluation of EBP on patient health outcomes, provider and patient satisfaction, and efficacy. As new knowledge is transformed through the five stages, the final outcome is EBP quality improvement of health care (Stevens, 2012).

This research project investigated the patient- and hospital-specific barriers to early hip fracture surgery in the older adult population. Exploration of these factors may lead to improved patient outcomes within this community hospital emergency department and subsequent integration into practice.

Next, the review of the literature will be presented. 


\section{Literature Review}

A literature search was undertaken using PubMed, Cochrane, Ovid, Medline Plus, NCBI, and Google Scholar databases. The key terms used were hip fractures, surgical timing/delay, patient-specific, comorbidities, and barriers to treatment, 30-day mortality, costs and standards for hip fracture care. Searches were limited to the English language, older adult population and human subjects. Studies conducted prior to the year 2000 were not included in this review. The term 'hip fracture' will be used throughout this research paper. It is a generalized term that encompasses all types of hip fractures including: Intraccapsular fracture, Intertrochanteric fracture, and Subtrochanteric fracture as well as femoral neck fracture, and neck of femur fracture (NOF).

\section{Introduction}

Hip fracture injuries are identified as one of the most serious healthcare problems affecting older adults (CDC, 2013). The prognosis after hip fracture is unfavorable and approximately $25 \%$ of elderly patients will die within one year after the event. Surgical repair is a critical element in the management of hip fracture. Research has shown that early surgical intervention has a lower 1-year mortality rate than those with surgical intervention at $2+$ days

\section{Mortality Risk after Hip Fracture}

One study assessed the two-year mortality rate after hip fracture in elderly patients by evaluating hospital factors such as: 1) intervention type; 2) surgical delay; and 3) patient-specific factors including underlying health conditions, lifestyle factors, body mass index, smoking and alcohol intake histories. The two-year mortality rate in hip 
fractures was associated with gender, age and comorbidities. Male gender, age older than 85 years, and higher comorbidity scores were associated with a higher risk of death (Castronuovo, Pezzotti, Franzo, Di Lallo, and Guasticchi, 2011). These findings were similar to a study of surgical delays, revealing early surgery reduces mortality risk in patients with numerous comorbidities (Meessen, et al, 2014).

The 30 day mortality rate after hip fracture surgery was examined in a tertiarycare hospital by Kahn, Hossain, Ahmed, Muthukumar and Mohsen (2013). The more common causes of mortality were pneumonia, acute coronary syndrome, and sepsis. Surgery performed 48 hours after admission had a significantly higher 30 mortality rate (11\% vs. $4 \%$ ). The strongest predictors of 30 day mortality were identified as surgical delay greater than 48 hours, admission source (other than home) and pre-existing cardiac disease.

An Italian study compared the effect of early surgery following hip fracture on one-year mortality of patients undergoing early surgery (within 48 hours) and patients undergoing surgery after 48 hours. A total of 405,037 cases of hip fracture were studied and the number of deaths prevented by early surgical intervention was 5,691. Patients who underwent surgery within two days had lower one-year mortality comparted to those who waited for surgery more than two days The study confirmed the association between delayed surgery and increased mortality and complication rates in elderly patients admitted after hip fracture. The data supported that deviating from surgical guidelines in hip fracture is costly, and may result in an increased mortality rate and prolonged hospital stay (Colais, Di Martino, Fusco, Perucci, \& Davoli, 2015). 
Early postoperative mortality predictors to reduce risk and improve outcomes after hip fracture in the older adult population have been identified (Raspopovic et al, 2015). Postoperative delirium was the only variable independently related to 30-day mortality after hip fracture in the older adult population. This finding is significant because postoperative delirium is one of the most common complications postoperatively. Older male patients with a lower cognitive status had a higher chance of developing postoperative delirium (Raspopovic et al.).

Campos et al. (2015) examined the one year survival rate and mortality predictors in patients with fracture of the proximal femur. Survival was assessed at three, six, nine and 12 months after fracture and repair. This data was compared to demographic factors, lifestyle and clinical history, as well as fracture type, surgery date, surgical treatment and perioperative risk. Two hundred fifty-two patients were studied (78.9\% were women). Mortality at three, six, and nine and 12 months was $21.2 \%, 25 \%, 28.8 \%$ and $34.6 \%$ for men and $7.8 \%, 13.5 \%, 19.2 \%$, and $21.4 \%$ for women. Each day of delay to surgery was associated with a $7 \%$ increased risk of death. Males were less likely than females to undergo surgery on the day of admission or the following day. This finding was thought to be the result of males having more comorbidities requiring longer time for stabilization, thus longer time to surgery. Predictors of death were male gender, American Society of Anesthesiologist (ASA) score, age, and delay in days to surgery. Furthermore, advanced age and male gender carried a higher risk of mortality after one year (Campos et al.). 


\section{Costs Associated with Hip Fracture}

Hospital value-based purchasing (VBP) aims to incentivize inpatient providers to deliver high value, as opposed to high volume health care. The formal mandate of hospitals to provide high value health care through financial incentives marks an important change in Medicare and Medicaid policy for reimbursement (Blumenthal \& Jena, 2013). Gu, Koenig, Mather, and Tongue (2014) asked the questions: (1) Are the societal benefits of hip fracture surgery enough to offset the direct medical costs? (2) Nationally, what are the total lifetime benefits of hip fracture surgery for a cohort of patients and to whom do these benefits accrue? They estimated the effects of surgical treatment for displaced hip fractures through a Markov cohort analysis. Data were collected from a systematic literature review, analysis of Medicare claims, and clinical experts. They estimated the average lifetime societal benefits per patient exceeded the direct medical costs of hip fracture surgery by $\$ 65,000$ to $\$ 68,000$ for displaced hip fractures

Judd and Christianson (2015) performed a retrospective chart review to determine whether patients who underwent early operative therapy (less than six hours after admission) versus later (more than six hours after admission) incurred decreased medical costs. The authors found the average cost of early intervention was $\$ 49,900$ compared with the average cost of later invention at $\$ 65,300$. There was no significant difference in the incidence of major complications between the two groups. However, one limitation to this study was identified as selection bias. Those patients who underwent early intervention may have had fewer comorbidities and needed less interventions than 
the group who underwent surgical intervention at the later time. This study suggested programs that emphasize expedited hip fracture surgery have the potential to produce significant healthcare savings and improved clinical outcomes (Judd \& Christianson).

\section{Delay to Surgery}

The Agency for Clinical Innovation (2014) recommended that all patients be assessed for medical stability in relation to perioperative risk. This risk should be documented, communicated, managed and minimized when possible. Optimizing time to surgery for patients with hip fracture requires multidisciplinary coordination between the emergency department (ED) and orthopedic, anesthetic and geriatric services.

Recommendations for patients deemed medically stable suggest surgery no more than 48 hours after admission (Agency for Clinical Innovation, 2014). The minimum standards for optimizing patient outcome after hip fracture according to the Agency for Clinical Innovation (2014) are as follows:

1. Older people with a hip fracture should be admitted under the joint responsibility of an orthopedic surgeon and a geriatrician

2. Optimal pain management

3. Surgery within 48 hours regardless of inter-hospital transfers.

4. Patient's surgery is not cancelled.

5. Commencement of mobilization with 24 hours of surgery.

6. Re-fracture prevention.

7. Local ownership of data systems processes to drive, improvements in care. 
Ryan, Yoshihara, Yoneoka, Egol, and Zuckerman (2015) aimed to defining an empirical threshold for delay of hip fracture surgery based on postoperative mortality and complication rates. Through a retrospective database analysis of hospital discharge data, a total of 2,121,215 patient's charts were reviewed. Logistic regressions were performed to assess the effect of surgical timing on in-hospital complication and mortality rates. When compared to same-day surgery, surgery two calendar days and three + days after admission was associated with higher mortality rates. Increased risk of complications for next-day surgery was OR: 1.09. This odds ratio became much greater for surgery 3 or more days after admission: OR: 2.08. Based on these findings, "delay" was defined as surgery performed 2 or more days after admission. (Ryan et al.)).

In a study conducted by Gomes et al. (2015), physicians explored whether age influenced the delay between diagnosis of hip fracture and surgical intervention. Onehundred and sixty-one adult patients were included in this study: 37 aged 17-59 years and 124 aged 60 and older. Among younger adults (ages 17-59), the mean delay was $6.4 \pm 5.3$ days; among those 60 and older, the delay was 9.5+7.6 days. Despite an increased susceptibility to complications caused by surgical delay, those patients aged 60 and older had the longest delays from admission to surgical treatment (Gomes et al.).

Patient-Specific Factors Contributing to Delay. Moran and colleagues (2005), conducted a prospective, observational study of 2660 patient who underwent surgical treatment to determine whether a delay in surgery for hip fractures affected postoperative mortality among elderly patients. The thirty-day mortality for patients for whom surgery had been delayed for more than 4 days was $10.7 \%$ and this group had 
significantly increased mortality at 90 days and one year. Patients who had acute medical comorbidities that required treatment prior to surgery, and had surgery delayed for more than 48 hours, had a thirty-day mortality of $17 \%$ which was nearly 2.5 times greater than those not requiring medical intervention prior to surgery. The researchers determined that 90 day mortality was not increased when the surgery was delayed up to four days for patients who were otherwise fit for hip fracture surgery, however, a delay of more than 4 days significantly increased mortality risk.

Bottle and Aylin (2006) examined data to estimate the number of deaths and readmissions associated with delay in surgery after femoral fracture. The design was an analysis of inpatient hospital episode statistics. There were 129,522 admissions for fractured neck of femur in 151 NHS hospital trusts in England, with 18,508 deaths in hospital. For all deaths in hospital the odds ratio for more than two days delay ranged between $1.1-62.4 \%$ between trusts. Only patients that were admitted from home were included in this study. Patients with comorbidities were more likely to experience a delay in surgical intervention. Bottle and Aylin concluded hip fracture patients are complicated and possess a plethora of comorbidities on presentation to the ED. In patients older than 60, a valid reason for delay was identified as the need to stabilize concurrent medical conditions. Some patient-specific factors identified as of concern included over-anticoagulation, anemia, volume depletion, electrolyte imbalances, uncontrolled diabetes or heart failure and correctable cardiac arrhythmia or ischemia (Bottle \& Aylin, 2006). 
Fantini et al. (2010), through a retrospective cohort study, analyzed factors contributing to delay in surgical treatment of hip fractures in older adult patients by more than two days. They further investigated whether these factors were consistent between a teaching and a community hospital. Both organizational and medical factors accounted for delays of surgical intervention. Potential patient and hospital specific predictors for surgical delay included gender, advanced age, hospital location, comorbidity, type of intervention (partial or total hip replacement vs. reduction and internal fixation), elevated international normalized ratio, decreased hemoglobin, ASA score and time between injury and admission.

Another patient-specific barrier to early surgery may be caused by delays related to the withdrawal of clopidogrel and the return of normal platelet function. Chechik et al. (2012) compared the morbidity and mortality rates in older adult patients receiving longterm treatment with clopidogrel who were operated on with or without delay secondary to withdrawal of clopidogrel following a hip fracture. They concluded that early surgical treatment of hip fractures in an elderly patient receiving long-term treatment with clopidogrel was safe in terms of decreased bleeding complications. The use of clopidogrel allowed for early ambulation and shorter hospital stay. The sample size of 60 patients resulted insufficient power to detect differences in mortality. However, patients in the delayed treatment group had more complications associated with immobilization. Data suggested clopidogrel needn't be discontinued prior to urgent hip fracture repair (Chechik et al.). 
Electrolyte imbalances have been identified as a patient-specific factor in delaying hip fracture repair. Aqil, Hossain, Sheikh, Aderinto, Whitwell and Kapoor (2015) aimed to identify medical risk factors of surgical delay, and assessed their impact on mortality. Biochemical parameters collected included admission hemoglobin, white blood cell count (WBC), coagulation profile, urea and electrolyte levels. Following a multivariate regression analysis, only hyponatremia was deduced to be a significant risk factor for delay. A Pearson chi-square test failed to demonstrate a statistical difference in mortality incidence between hyponatremic and normonatremic patients. The authors determined non-severe and isolated hyponatremia should not be a factor contributing to delay of surgery (Aqil et al).

Hospital-Specific Factors Contributing to Delay. A study conducted by Barone, Fusco, Colais, D’Ovidio, Belleudi, Agabiti, and Perucci, (2009) examined the association of patients' socioeconomic status with thirty-day mortality and delayed surgery after hip fracture. The data revealed a low socioeconomic level was significantly associated with a higher risk of mortality and wait time to surgery. Individuals living in disadvantaged communities had poorer prognoses and were less likely than more affluent individuals to be treated according to clinical guidelines despite universal healthcare coverage (Barone, et al.).

Dy, McCollister, Lubarsky, \& Lane (2011) examined systems-based factors contributing to hip fracture surgical delay. These factors included personnel cost, patient volume and patients receiving surgical treatment within 48 hours. Two potential 
strategies tabulating incremental cost and quality-adjusted life-years were created to evaluate cost-effectiveness). The first strategy focused on expediting preoperative evaluation by employing personnel outside regular 9-5 operating hours. The second strategy added an on-call team to staff the operating room outside of regular hours. The study found that systems-based solutions to minimize operative delay, such as a dedicated on-call support team, could be cost-effective to the ratio of $\$ 43,153$ per qualityadjusted life-years (Dy et al.).

Next, study methods will be presented. 


\section{Methodology}

\section{Purpose}

The purpose of this research project was to explore patient and hospital-specific barriers to early surgery for older adults with hip fractures in a community hospital setting

\section{Design}

This study was a retrospective chart review. Barriers to timely surgical repair of hip fracture in older adults was explored and identified.

\section{Sample}

The study sample consisted of medical records of patients aged 65 or older with an admission diagnosis of hip fracture. Inclusion criteria were records of patients of both genders, all racial and ethnic groups, and diverse cultural and socio-economic backgrounds. Exclusion criteria were records of patients with head injuries, associated pelvic fractures, patients who did not qualify for surgical intervention, and those patients requiring immediate interventions unrelated to hip fracture. Also excluded were patients who were transferred to another hospital, and one patient whose family decided on inpatient Hospice.

\section{Site}

The study was conducted at a 244-bed community hospital, part of a Lifespan system affiliated with Brown University. The project focused on the charts of patients who were 65 and older admitted through the ED with a diagnosis of hip fracture. 


\section{Procedures}

In March and April of 2017, the proposal was submitted for review and approval by the Institutional Review Boards (IRB) of the community hospital and Rhode Island College (RIC). After IRB approvals, data collection took place. The student researcher ran a report through Lifespan's electronic medical record system, EPIC. Medical record numbers of patients aged 65 and older diagnosed and admitted with hip fractures between January 1, 2016 and December 16, 2016 were collected.

During April of 2017, all electronic medical records were reviewed to identify inclusion and exclusion criteria. Data were collected in the ED in a non-patient care area using a computer that had access to the hospital's electronic health records. The ED office was accessed through punch code and badge access, which is only available to staff working in the ED. Electronic health records were accessed by a secure password and timed-out after 5 minutes when not in use to protect patient confidentiality. The time needed to review each record and tally the data was approximately 10 minutes. In April of 2017, data related to patient and hospital-specific delays to surgical repair of hip fractures in adults aged 65 and older was summarized.

\section{Measurement}

Quantitative data was collected using a data collection tool created by the researcher (Appendix B). The patient's age, gender, diagnosis, time of arrival in the ED, time of admission, time from admission to surgical intervention, comorbidities, medications on admission, current tobacco and alcohol use, type of surgical intervention, length of stay, and any identifiable patient or hospital-specific barriers to timely surgery 
were documented. Patient's age and gender was summarized in table format. Basic descriptive statistics were used to analyze the study variables. 


\section{Results}

A total of 120 records were reviewed. Twelve charts were excluded due to associated pelvic fractures. Seventeen records were excluded due to the date of fracture preceding the dates delineated in the research criteria. Seven records were excluded because the patient was transferred to another hospital and no further data was available. Eight records were excluded because the patient was below the age of 65 . Thirteen records were excluded because the fracture was determined to be inoperable by orthopedics, and one record was excluded because the family decided to transfer the patient to inpatient Hospice. Sixty-two records remained in the sample size. Of the total number of charts reviewed $(n=62), 88.7 \%(n=55)$ were female and $12.9 \%(n=8)$ were male.

The age and gender categories of the study subjects are illustrated in Figure 2.

\section{AGE GROUPS}

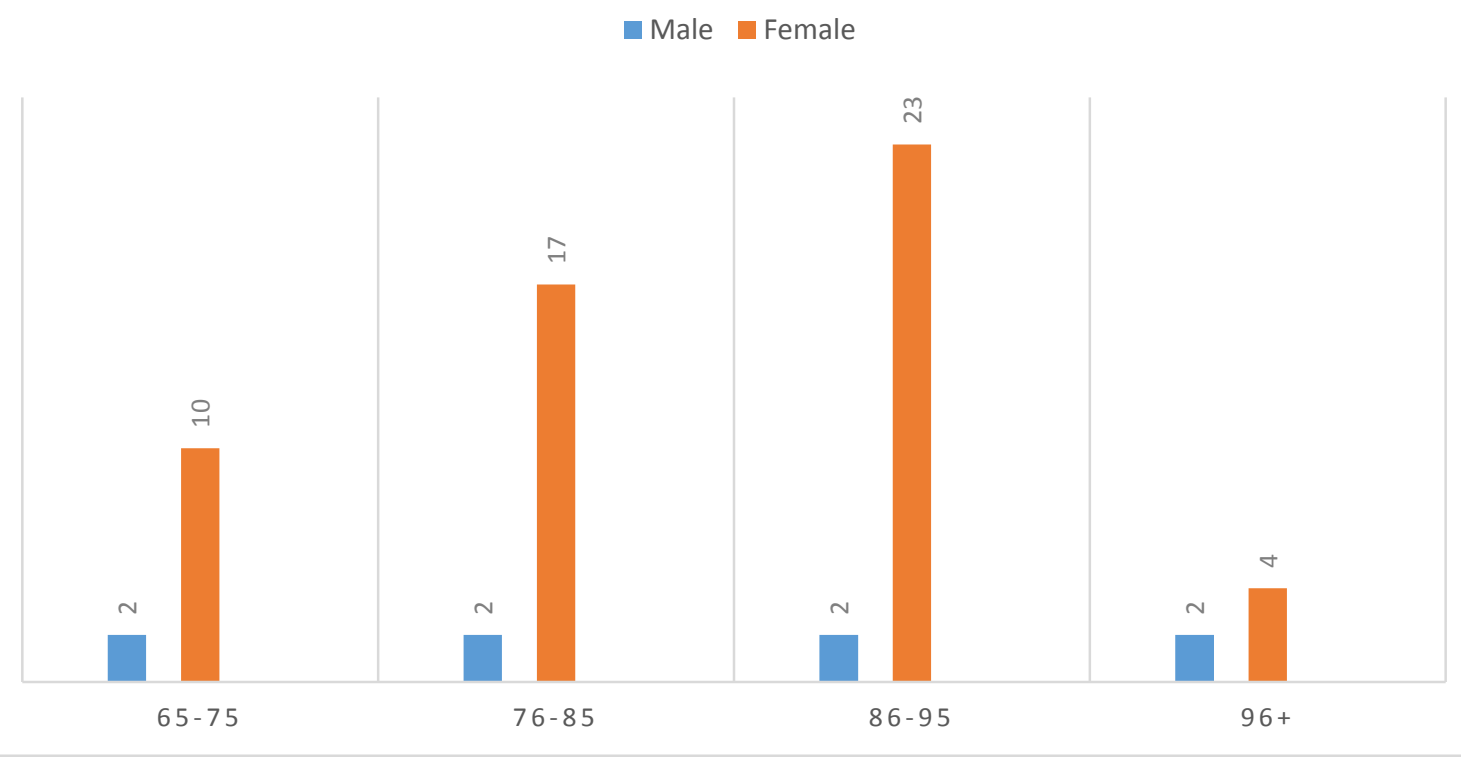


Figure 2. Age of study participants by gender

According to the literature, hip fractures effect women more often than men because men have higher bone density than women and because of the decrease in estrogen in women after menopause. The data in Figure 2 are consistent with these trends.

Average times from arrival to admission, admission to surgical intervention and length of stay are illustrated in Figure 3.

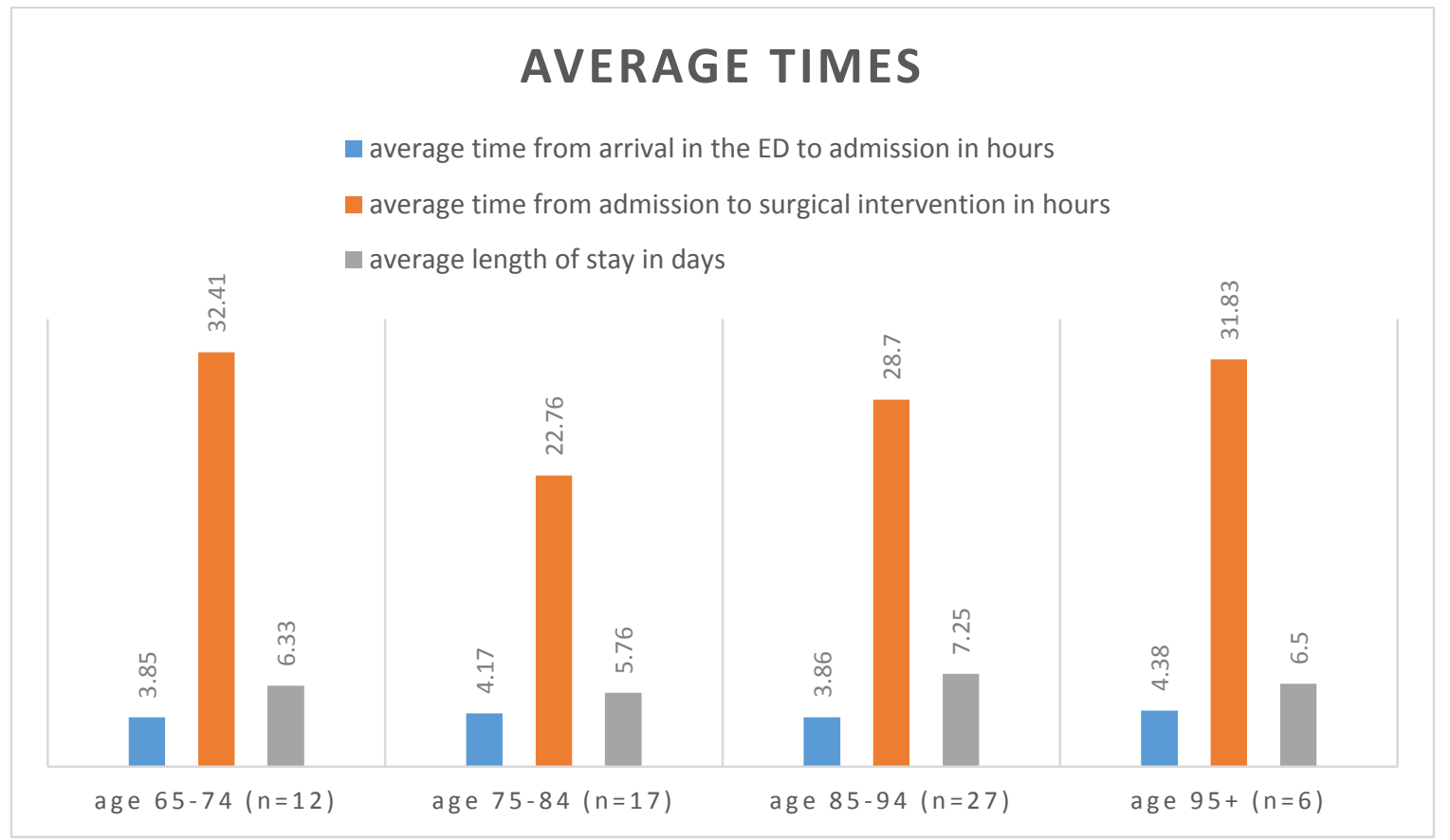

Figure 3. Average times for arrival to admission, admission to OR, and length of stay Time from arrival in ED to admission was 4 hours. Average time from admission to the operating room (OR) was 29 hours. Average length of stay was 6.5 days. 
The literature describes barriers to early surgery including over-anticoagulation and the need to withdraw clopidogrel to achieve normal platelet function. Because of these patient-specific factors identified, this researcher collected data related to patient medications including Xarelto, clopidogrel, Coumadin, Pradaxa, Plavix, and Aggrenox. One patient was on Xarelto, four patients were on Coumadin, one patient was on Pradaxa, four patients were on Plavix and one patient was on Aggrenox at the time of admission. Out of these 11 patients, one patient each on Aggrenox, Xarelto, Plavix, and Coumadin had a delay to surgical repair of hip fracture.

Diagnoses for patients included right and left hip fractures, intertrochanteric fractures, oblique introchanteric fractures, closed displaced oblique fractures, closed neck of femur fractures, comminuted proximal femur fractures, subcapital fractures, displaced subcapital fractures, displaced femoral neck fractures, nondisplaced femoral neck fractures, open type II femur fractures, and subtrochanteric fractures. Surgical interventions included; open reduction internal fixation of left and right hip fractures, cephalomeduallary nails, intramedullary rodding, gamma nail, hemiarthroplasty, partial hip arthroplasty, total hip arthroplasty, percutaneous pinning, total hip replacement, cemented hemi-arthroplasty, and open reduction percutaneous pinning. Data related to length of time for surgical intervention was not able to be obtained.

Eight charts were identified with a delay of more than 48 hours to surgical intervention. All of the charts identified belonged to females. 
Figure 4 shows the average number of comorbidities and average number of medications on admission for the patients who experienced a delay in hip fracture repair.



Figure4. Patients with delay to Surgical repair for Hip Fracture

The first record identified belonged to a 66 year old female with a right hip fracture. She had a past medical history of: HIV, polysubstance abuse, osteoarthritis, hypertension, anxiety, and Hepatitis C. Her medications on admission were; methadone, Xanax, Triumeq, Celebrex, Citalopram, Benadryl, and Lisinopril. She was a current every day smoker, but denied alcohol use. The patient arrived at 2000 and was admitted at 0100 the following morning. On 8/9 she was assessed by Orthopedics who cleared her for and recommended surgery, and clearly explained to patient that if she did not have surgery, she would not be able to walk again. According to the H\&P, patient "will not consider surgery." Patient was seen by immunology who documented, "patient wants to 
leave AMA, but cannot walk." Social work saw the patient on 8/9 and documented that the patient "plans on surgery," but when seen by Orthopedics later that same day, was refusing surgery again. On 8/10 at 0649 patient signed consent for surgery, but continued verbalizing that she did not want surgery. At 0816 it was documented that she was still refusing surgery. At 1147 there was documentation that the patient was being aggressive and abusive to staff. At 1211 the patient was evaluated by Psychiatry and deemed competent to make decisions. On $8 / 11$, the patient consented for surgery, and had an open reduction internal fixation of her right hip fracture. Patient had been admitted for 62 hours prior to having surgery.

The second record identified with a delay in surgical repair belonged to a 70 year old female with a past medical history including: coronary artery disease, a stroke within the previous 4 months, a pacemaker due to sick sinus syndrome, type II diabetes, COPD, dementia, GERD, hypercholesterolemia, hypertension, osteopenia, and peripheral vascular disease. Her medications on admission were; Aspirin, Lipitor, Symbicort, Metoprolol, Tums, Plavix, Lisinopril, and omeprazole. The patient arrived at the ED on $8 / 28$, but did not have surgical intervention until $8 / 31$. Patient had a cardiology consult on 8/29 with recommendations to continue Plavix. Possible reasons identified for delay identified were patient's extensive history of vascular disease, recent angioplasty on 8/11, and status post a left superficial femoral artery stent.

The third record identified belonged to a 77 year old female with a left hip fracture. She had a past medical history including congestive heart failure, hypertension, hyperlipidemia, nonspecific interstitial pneumonitis, diabetes, aortic stenosis, aortic 
insufficiency, hypothyroidism, and myocardial infarction. Her current medications included Amlodipine, Atenolol, Furosemide, Isosorbide, Imdur, Synthroid, Lisinopril, Metformin, pravastatin, Seroquel, and Ventolin. Patient arrived in the ED on 2/27 at 2107, was admitted on $2 / 28$ at 0015 , and had surgical intervention on $2 / 31$ at 1454 . In her History \& Physical, it was documented that there was evidence of mild acute diastolic heart failure. The patient was admitted to medicine for optimization prior to going to the OR. On 2/29 patient had an elevated troponin with no ischemic changes noted on EKG, and she was sent for an echocardiogram. Cardiology recommended that the patient was to "continue all medications", that "she may require additional diuretics post operatively", to "keep her hemoglobin above 10", and "in the absence of angina, significantly elevated troponin, or any significant wall motion abnormalities, she may proceed to the OR." There is no further documentation to indicate why the patient did not proceed to the OR on $2 / 29$ or $3 / 1$. The patient did not go to the OR for 62 hours after admission. One possible reason that should be considered was that the patient came to the ED on a Saturday.

The fourth record belonged to an 86-year-old female admitted for a right hip fracture. Her past medical history included hypertension and high cholesterol. Patient's medications on admission included: Aspirin, atenolol, atorvastatin, Lisinopril, Procardia, and a multivitamin. Patient arrived in the ER on $2 / 23$ at 0233 , was admitted on $2 / 23$ at 0924, and went to the OR on $2 / 25$ at 1937 . Patient went to the OR 58 hours after admission. Documentation indicated that the patient had tachycardia, high blood pressure and required hemodynamic stability. The patient was also non-English 
speaking.

The fifth record belonged to a 92-year-old female admitted for a left hip fracture. She had a past medical history including asthma, atrial fibrillation, breast cancer, hypertension, osteoporosis, Parkinson's, pleural effusion and transient ischemic attack. Patient's current medications on admission included; Arimidex, Aspirin, Sinemet, Vitamin D3, Levothyroxine, refresh dry eye, sennosides, and Xarelto. Patient arrived in the ED on $9 / 11$ at 1428 , was admitted on $9 / 11$ at 1811 , and went to the OR on $9 / 13$ at 2349. Patient's Xarelto was held on 9/11, and Hospice was considered by the patient's family. On 9/12 patient was found to have a distal deep vein thrombosis (DVT). On 9/13, patient was an add-on for surgery. Time from admission to OR was 60 hours.

The sixth record identified belonged to a 93-year-old female admitted for left hip fracture. Her past medical history included coronary artery disease, cardiac stents, peripheral vascular disease, lower extremity stents, congestive heart failure, diabetes, hypertension, atrial fibrillation, osteoarthritis, and peripheral artery disease. Patient medications on admission included; Coumadin, aspirin, valsartan, vitamin D, Plavix, Lasix, insulin and metoprolol. Patient arrived in the ED on Friday 8/5 at 1828, was admitted on $8 / 5$ at 2302 , and went to the $\mathrm{OR}$ on $8 / 8$ at 1656 . A documented Anesthesiologist's (on call) note recommended "surgery during daylight hours on Monday secondary to needing cardiologist or interventional radiologist's assist should any stents fail." A cardiology consult referred to a recent drug eluding stent to the left anterior tibial artery. Their recommendation was to continue Plavix. The patient also needed to receive two units of packed red blood cells due to anemia. Time from 
admission to OR was 66 hours.

The seventh record belonged to a 94-year-old female with a past medical history including anemia, asthma, hypokalemia, hypertension, syncopal episodes, thyroid disease, clostridium difficile infection, transient ischemic attack, anxiety, depression, and stroke. Her medications on admission included; Tylenol, calcium carbonate, Klonopin, Aricept, Vasotec, Vitamin D, lactobacillus, Synthroid, lidocaine patch, Imodium, Lopressor, Pro air, Zoloft, and Aggrenox. Patient arrived in the ED on 12/12 at 0912, was admitted 12/12 at 1111, and went to the OR 12/14 at 2052, 58 hours after admission. When patient was in the ED, she was found to have a hemoglobin of seven, and subsequently received 2 units of packed red blood cells. In pre-op on 12/13, patient was noted to have rales and needed to be diuresed. There was clear documentation by Orthopedics that they would be available for surgery when the patient was stable.

The eighth and last record identified belonged to a 98-year-old female admitted with a right hip fracture. She had a past medical history of coronary artery disease, nonST elevation myocardial infarction (NSTEMI), pneumonia, hypertension, hyperlipidemia, depression, and congestive heart failure (CHF). Her medications included amlodipine, vitamin D, psyllium packets, trazadone, Tylenol, citalopram, Miralax, aspirin, furosemide, and omeprazole. Patient arrived in the ED on 2/9 at 0546, was admitted 2/9 0817, and went to the OR on 2/11 at 1700, 59 hours after admission. Possible reasons for delay were "severe hypertension," and "acute anemia."

Current tobacco and alcohol use was inconsistently documented throughout all 62 records. Tobacco use has been proven to hold a detrimental effect on wound healing and 
would therefore be an important piece of data to collect from the patient. Smoking cessation counseling should always be offered to patients admitted to a hospital.

Likewise, alcohol and its overuse can cause serious harm to the patient. If alcohol use is not elicited from the patient, and indeed the patient has a problem with alcohol, very serious effects from withdrawal can occur to the patient while in the hospital, including delirium tremens, and even death.

Summary and conclusions will be presented next. 


\section{Summary and Conclusions}

A hip fracture is a debilitating condition that consumes significant resources in the United States. Surgical treatment of hip fractures can achieve better survival and functional outcomes than non-operative treatment (Gu, Koenig, Mather, \& Tongue 2014). Optimizing time to surgery for patients with hip fracture requires multidisciplinary coordination between the emergency department (ED) and orthopedic, anesthetic and geriatric services. Recommendations for patients deemed medically stable suggest surgery no more than 48 hours after admission (Agency for Clinical Innovation, 2014).

Patients with comorbidities are more likely to experience a delay in surgical intervention. Bottle and Aylin (2006) concluded hip fracture patients are complicated and possess a plethora of comorbidities on presentation to the ED. In patients older than 60 , a valid reason for delay was identified as the need to stabilize concurrent medical conditions. Some patient-specific factors identified as of concern included overanticoagulation, anemia, volume depletion, electrolyte imbalances, uncontrolled diabetes or heart failure and correctable cardiac arrhythmia or ischemia (Bottle \& Aylin, 2006).

The purpose of this research project was to explore and identify patient and hospital-specific barriers to early surgery for older adults with hip fractures in a community hospital setting. The study was a retrospective chart review. A total of 120 records were reviewed and 62 records remained in the sample. Of the total number of charts reviewed $(n=62), 88.7 \%(n=55)$ were female and $12.9 \%(n=8)$ were male.

Quantitative data were collected using a data collection tool created by the researcher (Appendix B). The patients' age, gender, diagnosis, time of arrival in the ED, 
time of admission, time from admission to surgical intervention, comorbidities, medications on admission, current tobacco and alcohol use, type of surgical intervention, length of stay and patient and hospital-specific barriers to timely surgery were documented.

Eight charts were identified as having a delay to surgery, the majority of which were determined to be patient-specific factors causing delay. These delays were as follows: "the patient would not consider surgery," "patient wants to leave against medical advice (AMA) but cannot walk," "patient with extensive history of vascular disease," "recent angioplasty," "elevated troponin," "mild acute diastolic heart failure," "tachycardia," "high blood pressure," "family is considering Hospice," "DVT," "anemia requiring two units of blood," "rales noted in pre-op requiring diuresis," "severe hypertension," and "anemia."

Possible hospital-specific delays deduced included a patient admitted on a Saturday, one on-call anesthesiologist's note recommending, "surgery during daylight hours on Monday secondary to needing cardiologist or interventional radiologist's assist should any stents fail," and one patient who had a delay due to "severe hypertension," was also Spanish speaking only. It is not clear in the documentation if this was a factor contributing to delay to surgery. There was no documentation regarding surgeries being cancelled for this population.

The study was limited by the relatively small sample size, unequal number of male and female participants and its' retrospective nature. Data related to delays to surgery had to be deduced from documentation. There was inconsistency in 
documentation related to time of incision for surgery. Approximately half of the charts examined had time of incision clearly documented. For the other half, this researcher used the time of the operative note. This note could have been written immediately after the patient's surgery, but may not have been. Although several patient-specific possible reasons for delay were clearly documented, there were only minor hints at hospitalspecific factors related to delay.

Although inclusion criteria for study included records of patients of all racial and ethnic groups, and diverse cultural and socio-economic backgrounds, specific data related to these criteria were not collected. In only one record was there mention of a patient being "Spanish speaking only." Information related to patient's insurance status or socioeconomic background was not collected. Data regarding current tobacco and alcohol use was poorly documented and inconsistent in nearly all charts. Costs associated with hip fractures were not gathered. When taking into consideration the minimum standards for optimizing patient outcome after hip fracture, optimal pain management was not examined. Other limitations in this study were that the researcher did not consider if the patient had been admitted under the joint responsibility of an orthopedic surgeon and a geriatrician and no documentation could be found regarding hip fracture surgeries being cancelled. Data related to early mobilization was not included in this project, and would be valuable information to gather in further research. Re-fracture prevention teaching and data related to three, six, and 12 month mortality rates after hip fracture would also be valuable information to obtain. 
Based on this research, some conclusions can be made. Documentation regarding time to incision was inconsistent. Although the average time from admission to surgical intervention was determined to be 29 hours, it may have actually been less than that. For those charts that did not have a specific time noted for time of incision, operative time had to be deduced by this researcher to be at the time of the operative note, which may have been several hours after the surgery took place. Consistent documentation is crucial for maintaining accurate records for patients undergoing surgery. The time documented could mean the difference between a patient receiving care according to the standards or not. If it is not documented, it is not done.

Pain management and early mobilization in relation to hip fractures was not collected. Older patients are notoriously under medicated for pain. This data would be invaluable to obtain for future research. The topic of hip fracture should have continued attention in advanced practice, in education, policy change, and on-going research. Research should be ongoing to promote best practice. Additional quality improvement projects could enhance clinical nursing practice and improve patient outcomes

It can be concluded based on the data collected that this community hospital is meeting expectations for early surgical intervention based on the Agency for Clinical Innovation minimum standards for optimizing patient outcome after hip fracture. Recommendations and implications for advanced practice will be presented next. 


\section{Recommendations and Implications for Advanced Nursing Practice}

Advanced practice nurses (APRN) are in a strong position to influence policy and standards by using evidence-based research. By staying current with the literature and continuing to be involved in research, the APRN continues to stand out as leader involved in developing best practice policies and guidelines. In collaboration with other members of the interdisciplinary team, the APRN can write policy and be a change agent in their facility. The APRN is a leader and should be knowledgeable about current health care policies not only in their own institution, but also at the state and federal level. Keeping current allows the APRN to lobby for change and reform.

The APRN possesses the qualities of an educator, a mentor, and a role model. APRNs have the opportunity to identify knowledge gaps in clinical practice. The APRN leads by example, applies the standard in practice, and influences others to follow any established protocols aimed at optimizing the patient flow logistics and management of comorbidities. This is crucial to make hospitals more patient-centered and to improve patient outcomes. APRNs work in EDs nationwide and are continually on the front lines caring for patients with hip fractures. As our population continues to increase in age, APRNS can advocate improving patient quality measures for early surgical intervention for hip fracture patients. The APRN guides and educates staff towards the most appropriate interventions and best practices for these patients, providing for a more competent, effective, and safe patient experience. Continuing education for the APRN is vital. The APRN should remain up to date on evidence based practice and research treatments for the management of hip fractures. 
Protocols aimed at optimizing patient outcomes include: Patient identification - at risk patient is identified at entry to health system, or other intervention points such as outpatient, community and primary care settings. Care coordination - Patient is referred for care coordination/case management through Fracture Liaison Coordinators to support access to appropriate investigation and medical care. Access to investigations and medical care - All patients who have a minimal trauma fracture should be assessed for appropriate medical therapy to decrease further risk of fractures. Care pathways Patients should then progress through care pathways that address: follow-up medical checks, chronic care interventions (including disease management); behavior changes, and access to community services, including falls prevention, aged care services and allied health (Agency for Clinical Innovation, 2014, p. 17).

Continuing research in the field of hip fractures is crucial to the well-being of any patient or family member involved. Because hip fractures are so costly, it is also crucial to healthcare facilities to manage these patients in the most efficient manner possible. In order to optimize patient and hospital outcomes, further research exploring: joint responsibility of an orthopedic surgeon and a geriatrician, optimal pain management techniques, surgery within 48 hours regardless of inter-hospital transfers, patient's surgery not being cancelled, commencement of mobilization with 24 hours of surgery, and re-fracture prevention strategies, is indicated. 


\section{References}

Agency for Clinical Innovation. (2014). Minimum standards for the management of hip fracture in the older person. Retrieved from http://www.aci.health.nsw.gov.au

American Academy of Orthopedic Surgeons. (2014). Management of hip fractures in the elderly evidence-based clinical practice guideline. Retrieved from http://www.aaos.org/cc_files/aaosorg/research/guidelines/hipfxguideline.pdf

Aqil, A., Hossain, F., Sheikh, H., Aderinto, J., Whitwell, G., \& Kapoor, H. (2015). Achieving hip fracture surgery within 36 hours: an investigation of risk factors to surgical delay and recommendations for practice. L Orthopaed Traumatol. doi.org/10.1007/s10195-015-0387-2

Barone, A. P., Fusco, D., Colais, P., D’Ovidio, M., Belleudi, V., Agabiti, N., Perucci, C. A. (2009). Effects of socioeconomic position on 30-day mortality and wait for surgery after hip fracture. International Journal for Quality in Health Care, 21, 379-386. doi.org/10.1093/intqhe/mzp046

Blumenthal, D., \& Jena, A. B. (2013). Hospital value-based purchasing. Journal of Hospital Medicine, 8, 271-277. doi.org/10.1002/jhm.2045

Bottle, A., \& Aylin, P. (2006). Mortality associated with delay in operation after hip fracture: observational study. Online First bmj.com, 1-5. doi.org/10.1136/bmj.38790.468519.55

Campos, S., Ferreira Alves, S. M., Carvalho, M. S., Neves, N., Trigo-Cabral, A., \& Pina, M. F. (2015). Time to death in a prospective cohort of 252 patients treated for 
fracture of the proximal femur in a major hospital in Portugal. Cad. Saude Publica, 7, 1528-1538. doi.org/org/10.1590/0102-311X00077714

Castronuovo, E., Pezzotti, P., Franzo, A., Di Lallo, D., \& Guasticchi, G. (2011). Early and late mortality in elderly patients after hip fracture: a cohort study using administrative health databases in the Lazio region, Italy. BMC Geriatrics, 11(37), 1-9. Retrieved from http://www.biomedcentral.com/1471-2318/11/37

CDC.gov. (2013). Retrieved from http://www.cdc.gov/features/agingandhealth/state of aging and health in America 2013.pdf

CDC.gov. (2016). Retrieved from http://www.cdc.gov/homeandrecreationalsafety/falls/fallcost.html

Chechik, O., Amar, E., Khashan, M., Kadar, A., Rosenblatt, Y., \& Maman, E. (2012). In support of early surgery for hip fractures sustained by elderly patients taking Clopidogrel. Drugs Aging, 29(1), 63-68. http://dx.doi.org/10.2165/11598490

Colais, P., Di Martino, M., Fusco, D., Perucci, C. A., \& Davoli, M. (2015). The effect of early surgery after hip fracture on 1-year mortality. BMC Geriatrics, 15(141), 1-8. doi.org/10.1186/s12877-015-0140-y

Dy, C. J., McCollister, K. E., Lubarsky, D. A., \& Lane, J. M. (2011). An economic evaluation of a systems-based strategy to expedite surgical treatment of hip fractures. The Journal of Bone and Joint Surgery, Incorporated, 93, 1326-1334. doi.org/10.2106/JBJS.I.01132

Fantini, M. P., Fabbri, G., Laus, M., Carretta, E., Mimmi, S., Franchino, G, Rucci, R. (2010). Determinants of surgical delay for hip fracture. The Surgeon, Journal for 
the Royal Colleges of Surgeons of Edinburgh and Ireland, 9, 130-134.

doi.org/10.1016/j.surge.2010.11.031

Forni, S., Pieralli, F., Sergi, A., Lorini, C., Bonaccorsi, G., \& Vannucci, A. (2016).

Mortality after hip fracture in the elderly: The role of a multidisciplinary approach and time to surgery in a retrospective observational study on 23,973 patients. Archives of Gerontology and Geriatrics, 66, 13-17. doi.org/.org/10.1016/j.archger.2016.04.014

Gomes, L. P., Do Nascimento, L. D., Campos, T. V., Paiva, E. B., Percope De Andrade, M. A., \& Guimaraes, H. C. (2015). Influence of age on delayed surgical treatment of proximal femoral fractures. Acta Ortop Bras, 23, 315-318.

doi.org/org/10.1590/1413-785220152306149049

Gu, Q., Koenig, L., Mather, R. C., \& Tongue, J. (2014). Surgery for hip fracture yields societal benefits that exceed the direct medical costs. Clinical Orthopedics and Related Research, 472, 3536-3546. doi.org/10.1007/s11999-014-3820-6

Judd, K. T., \& Christianson, E. (2015). Expedited operative care of hip fractures results in significantly lower cost of treatment. The Iowa Orthopedic Journal, 35, 62-64.

Kahn, M. A., Hossain, F. S., Ahmed, I., Muthukumar, N., \& Mohsen, A. (2013). International Orthopedics, 37, 2119-2124. doi.org/10.1007/s00264-013-2068-1

Meessen, J. M., Pisani, S., Gambino, M. L., Bonarrigo, D., Van Schoor, N. M., Fozzato, S., Surace, M. F. (2014). Assessment of mortality risk in elderly patients after proximal femoral fracture. Orthopedics, 194-200. doi.org/10.3928/0147744720140124-25 
Moran, C. G., Wenn, R. T., Sikand, M., \& Taylor, A. M. (2005). Early mortality after hip fracture: Is delay before surgery important? The Journal of Bone and Joint Surgery, 87-A, 483-489. doi.org/10.2106/JBJS.D.01796

Orosz, G. M., Magaziner, J., Hannan, E. L., Morrison, R. S., Koval, K., Gilbert, M., Siu, A. L. (2004). The timing of surgery for hip fracture and its effects on outcomes. JAMA, 291, 1738-1743. doi.org/ 10.1001/jama.291.14.1738

Raspopovic, E. D., Denic, L. M., Marinkovic, J., Radinovic, K., Ilic, N., Vujadinovic, S. T., \& Kadija, M. (2015). Early mortality after hip fracture: what matters? Psychogeriatrics, 15, 95-101. doi.org/10.111/psyg.12076

Ryan, D. J., Yoshihara, H., Yoneoka, D., Egol, K. A., \& Zuckerman, J. D. (2015). Delay in hip fracture surgery: An analysis of patient-specific and hospital-specific risk factors. Journal of Orthopedic Trauma, 29, 343-348.

Salkeld, G., Cameron, I. D., Cumming, R. G., Easter, S., Seymour, J., Kurrle, S. E., \& Quine, S. (2000). Quality of life related to fear of falling and hip fracture in older women: a time trade off study. British Medical Journal, 320, 341-346.

Stevens, K. R. (2012). Star Model of EBP: Knowledge Transformation. Retrieved from The University of Texas Health Science Center at San Antonio: http://www.nursing.uthscsa.edu 
Appendix A

ACE Star Model of Knowledge Transformation

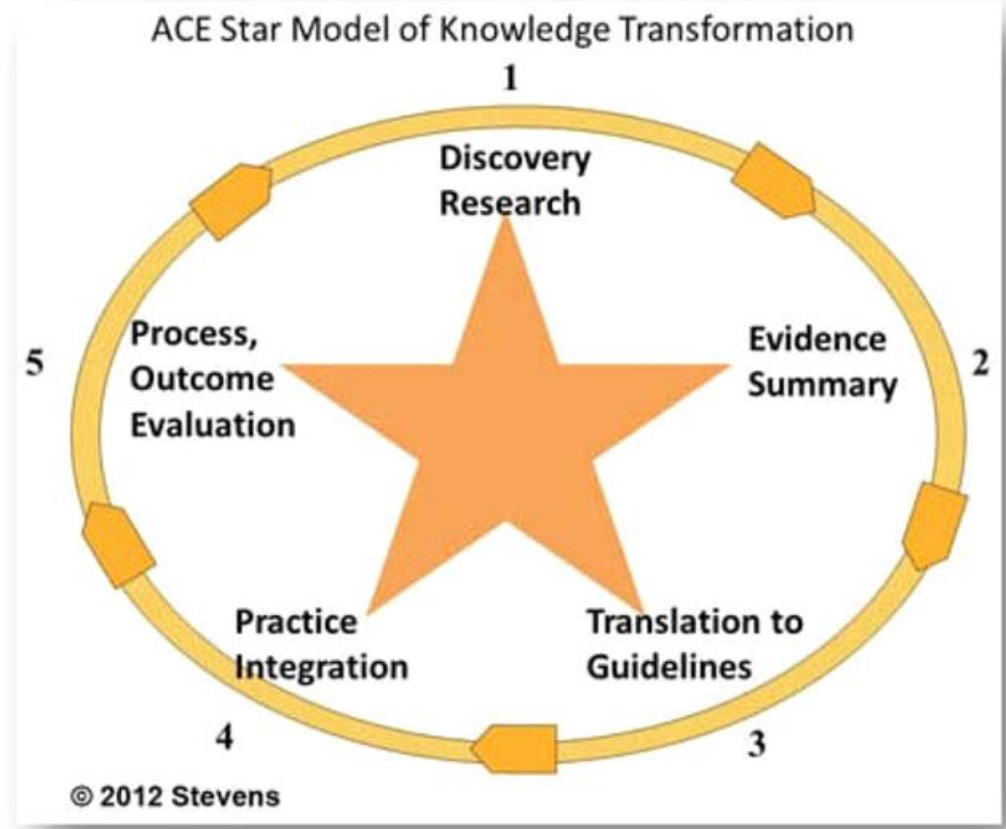

Stevens, K. R. (2012). Star Model of EBP: Knowledge Transformation. Retrieved from The University of Texas Health Science Center at San Antonio:

http://www.nursing.uthscsa.edu 
Appendix B

Data collection tool

Date

age

gender

diagnosis

ED time

time of admission

time of incision/Op note

Comorbidities

Medications on admission

Current Tobacco/Alcohol use

Type of surgical intervention

Discharge date

Delays identified? 